\title{
Stabilizing Second-Order Linear Dynamic Systems Via Hybrid Output Feedback Controls
}

\author{
Liguo Zhang, Yangzhou Chen, and Pingyuan Cui \\ School of Electronic and Control Engineering, \\ Beijing University of Technology,Beijing, 100022, China \\ zhangliguo@emails.bjut.edu.cn
}

\begin{abstract}
This paper considers the open problem whether there exists a finite-state hybrid output feedback control to asymptotically stabilize a second-order linear dynamic system. More precisely, for secondorder linear time-invariant systems which are not stabilizable via a single static output feedback, we find two different output feedback gains and a switching law orchestrating the feedback gains such that the closed-loop system is asymptotically stable.
\end{abstract}

\section{Introduction}

Given a linear time-invariant dynamic system

$$
\begin{aligned}
\dot{x}(t) & =A x(t)+B x(t) \\
y(t) & =C x(t)
\end{aligned}
$$

where $x \in R^{n}, u \in R^{m}, y \in R^{p}$, and $A, B, C$ are matrices of suitable dimensions, if system (1) is stabilizable and detectable, then there exists a linear dynamic output feedback law that asymptotically stabilizes the system [1]. In practice, however, such continuous dynamic feedback might not be implemental, and a hybrid version of the controller is often desired. We present a motivation example, which was also discussed in [2], 3] and [4].

Example 1. Consider the harmonic oscillator model, which can be written in the form of (1) with

$$
A=\left(\begin{array}{cc}
0 & 1 \\
-1 & 0
\end{array}\right), \quad B=\left(\begin{array}{l}
0 \\
1
\end{array}\right), \quad C=\left(\begin{array}{ll}
1 & 0
\end{array}\right) .
$$

Although this system is both controllable and observable, it cannot be stabilized by a single static output feedback [z] ; however, it is stabilizable by a hybrid output feedback [3], [4]. Letting $u=-y$ and $u=\frac{y}{2}$ we obtain the following systems, respectively,

$$
\dot{x}(t)=\left(\begin{array}{cc}
0 & 1 \\
-2 & 0
\end{array}\right) x(t)
$$

and

$$
\dot{x}(t)=\left(\begin{array}{cc}
0 & 1 \\
-\frac{1}{2} & 0
\end{array}\right) x(t) .
$$


Define $V(x)=x_{1}^{2}+x_{2}^{2}$. If system (3) is active in the first and third quadrants, while system (4) is active in the second and fourth quadrants, we will have $\dot{V}<0$ whenever $x_{1} x_{2} \neq 0$, which implies that the entire switched system is asymptotically stable by LaSalle's Principle (e.g., [15]).

Therefore, the hybrid output feedback stabilization problem can be formulated as follows [5]: find a finite collection of $m \times p$ gain matrices $\left\{K_{1}, \cdots, K_{l}\right\}$, and a switching law $\sigma:[0, \infty) \rightarrow\{1, \cdots l\}$, such that the switched linear system $\dot{x}(t)=A_{\sigma(t)} x(t)$ is asymptotically stable, where $A_{\sigma(t)}=A+B K_{\sigma(t)} C$, and the output feedback control is given by $u(t)=K_{\sigma(t)} y(t)$. To rule out trivial cases, we assume the system (1) cannot be static output feedback stabilized.

Stability analysis for switched systems has achieved many results, which mostly based on the common Lyapunov function or the multiple Lyapunov function approach [6], [7, [8], [9, [10, 11. Stabilization problem is the control design for stability, while available techniques that can be used in the hybrid output feedback control have been very few. The difficulty led researchers to study it first in the low-order systems. In [12, it has been shown that if system (1) is controllable and observable, then it admits a stabilizing hybrid output feedback that uses a countable number of discrete states. More recently, [5] constructs a 2-state output feedback for a class of second-order linear systems incorporated with a conic switching law [13, [14, which provide a description of the switching law based on the angles of subsystem vector fields and the geometric properties of the phase plane. However, their approach may be complicated to work with or determine the exact parametric value for which the switching law yields instability of the feedback system.

In this paper, we propose an approach for constructing a 2-state hybrid output feedback control to stabilize a second-order linear dynamic system. This approach is composed of two important parts. First, we consider the asymptotic stabilization problem of second-order autonomous switched linear systems consisting of two subsystems with unstable focus equilibrium. Using the "most stabilizing" switching law, we translate the switched system into a piecewise linear system that yields an easily verifiable sufficient condition for stabilization of this system. Secondly, 2-state output feedback gains are constructed in terms of control system matrices $(A, B, C)$, which extends the results in [5].

The rest of the paper is organized as follows. Section 2 analyzes the asymptotic stabilization for an autonomous switched linear system. Section 3 designs the 2state hybrid output feedback control. Numerical examples are given in section 4. Section 5 summarizes.

\section{Stabilization for Switched Linear Systems}

Consider an autonomous switched linear system given by

$$
\begin{aligned}
& \dot{x}(t)=A_{\sigma(t)} x(t) \\
& \sigma:[0, \infty) \rightarrow\{1,2\}
\end{aligned}
$$


where $x \in R^{2}$ and $\sigma(t)$ is the switching law indicating the active subsystem at each instant. Let $\lambda_{i, \pm}=\alpha_{i} \pm \beta_{i}$ be the eigenvalues of $A_{i}$, respectively.

Lemma 1. [16]. For the two subsystems of the switched linear system (5), there exists a nonsingular matrix $Q$, such that

$$
Q A_{1} Q^{-1}=\left(\begin{array}{cc}
\alpha_{1} & \beta_{1} / E \\
-E \beta_{1} & \alpha_{1}
\end{array}\right), \quad Q A_{2} Q^{-1}=\left(\begin{array}{cc}
\alpha_{2} & \beta_{2} \\
-\beta_{2} & \alpha_{2}
\end{array}\right) .
$$

Without loss of generally, we only discuss the results for $|E|<1$. In the case of $0<E<1$, the system trajectory diverges to $\infty$ clockwisely, while in the case $-1<E<0$, then counter-clockwisely. Therefore, in the following discussion, we assume the subsystems' state matrices have the form (6).

We first observe that the locus of both subsystems circle around the origin and diverge to clockwisely or counter-clockwisely. We will show that the "most stabilizing" switching law corresponds to switching between the two subsystem's vector fields are parallel. Here, by "most stabilizing" case, we mean if the switched system is stabilizable, then the system's trajectory converges to the origin under this switching law, that is, select an active subsystem which would drive the trajectory closer to the origin.

Define $l_{-}$and $l_{+}$as the lines on which the subsystem's vector field $A_{1} x$ and $A_{2} x$ are parallel. Then on $l_{ \pm}$we have

$$
\frac{-\beta_{2} x_{1}+\alpha_{2} x_{2}}{\alpha_{2} x_{1}+\beta_{2} x_{2}}=\frac{-E \beta_{1} x_{1}+\alpha_{1} x_{2}}{\alpha_{1} x_{1}+(1 / E) \beta_{1} x_{2}} .
$$

Solving this equation we obtain that if

$$
D \geq 0
$$

then

$$
x_{2}=\nu_{ \pm} x_{1},
$$

where, when

$$
\rho_{1}-\frac{\rho_{2}}{E} \neq 0,
$$

we have,

$$
\nu_{ \pm}=\frac{(E-(1 / E)) \mp 2 \sqrt{D}}{2\left(\rho_{1}-\left(\rho_{2} / E\right)\right)}
$$

or, when

$$
\rho_{1}-\frac{\rho_{2}}{E}=0,
$$

we have,

$$
\begin{gathered}
\nu_{-}=\infty \\
\nu_{+}=\frac{\rho_{1}-E \rho_{2}}{E-(1 / E)} .
\end{gathered}
$$

We denote

$$
D=R^{2}+2 \rho_{1} \rho_{2} R-\left(1+\rho_{1}^{2}+\rho_{2}^{2}\right)
$$


and

$$
R=\frac{1}{2}\left(E+\frac{1}{E}\right), \quad \rho_{1}=\frac{\alpha_{1}}{\beta_{1}}, \quad \rho_{2}=\frac{\alpha_{2}}{\beta_{2}} .
$$

Define the regions

$$
\begin{gathered}
R_{1}=\left\{\left(x_{1}, x_{2}\right) \mid \nu_{-} x_{1}>x_{2}>\nu_{+} x_{1}\right\}, \\
R_{2}=\left\{\left(x_{1}, x_{2}\right) \mid x_{2} \geq \nu_{-} x_{1} \text { and } x_{2} \geq \nu_{+} x_{1}\right\},
\end{gathered}
$$

where $-R_{i}$ is the opposite region of $R_{i}$ against the origin on the phase plane, $i=1,2$, see Fig. 1 .

In the case $0<E<1$, the trajectories of subsystems 1, 2 are all clockwise logarithmic spirals. Then the "most stabilizing" switching law would be:

Switch to subsystem 1 whenever the system trajectory enters the regions $R_{1}$ and $-R_{1}$, and switch to subsystem 2 whenever the system trajectory enters the regions $R_{2}$ and $-R_{2}$.

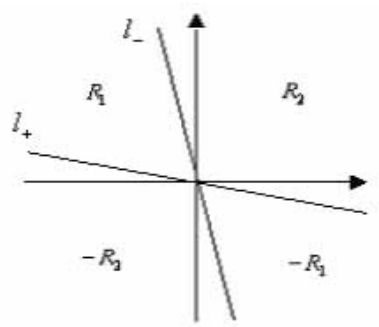

Fig. 1. Region partition when $D>0$

Under the "most stabilizing" switching law, we have translated system (5) into a piecewise linear system

$$
\dot{x}=\left\{\begin{array}{ll}
A_{1} x & x \in R_{1} \cup-R_{1} \\
A_{2} x & x \in R_{2} \bigcup-R_{2}
\end{array} .\right.
$$

Now, if the piecewise linear system (11) is asymptotically stable, then the switched linear system (5) asymptotically stabilizable.

Theorem 1. [16]. The autonomous switched linear system (5) consisting of two subsystems with unstable focus equilibrium is asymptotically stabilizable if $D>0$, $0<E<1$ and $\rho<1$, where

$$
\begin{aligned}
\rho=\exp & {\left[\rho_{1} \arctan \left(\frac{\rho_{1} R-\rho_{2}}{\sqrt{D}}\right)+\rho_{2} \arctan \left(\frac{\rho_{2} R-\rho_{1}}{\sqrt{D}}\right)-\frac{\pi}{2}\left(\rho_{1}+\rho_{2}\right)\right] } \\
\times \sqrt{\frac{\left(\rho_{1} \rho_{2}+R\right)+\sqrt{D}}{\left(\rho_{1} \rho_{2}+R\right)-\sqrt{D}}} &
\end{aligned}
$$


Remark 1. For $D>0,0<E<1$, the trajectories of subsystems 1,2 are counterclockwise, clockwise logarithmic spirals, respectively. In the case we could also define the "most stabilizing" switching law and get the similar conclusion as Theorem 1 . When $D>0$, then at least on the half phase plane, one subsystem's vector field always points on the same side of other. Form the proof of Theorem 1 we know in this case system (5) cannot be asymptotically stabilizable.

\section{Hybrid Output Feedback Control}

In this section, we construct 2-state hybrid output feedback gains in terms of control system matrices $(A, B, C)$, which deduced closed-loop system matrices satisfy the conditions of Theorem 1. For simply, we only focus discussion on second-order single-input-single-output (SISO) systems, i.e., $m=2, n=p=1$ in system (1). Since a nonsingular linear transformation does not change the stabilization property, we consider system (1) in the following form:

$$
A=\left(\begin{array}{ll}
a_{11} & a_{12} \\
a_{21} & a_{22}
\end{array}\right), \quad B=\left(\begin{array}{l}
0 \\
1
\end{array}\right), \quad C=\left(\begin{array}{ll}
1 & c
\end{array}\right),
$$

where $a_{i j}, c \in R, i, j=1,2$.

Theorem 2. For the second-order linear time-invariant system (13), if $c=0$, and $a_{12} \neq 0$, then it is stabilizable via a static or 2-state hybrid output feedback.

Proof. For system (13), the closed-loop system with output feedback $u=k_{i} y$ is given by $\dot{x}=A_{i} x, i=1,2$, where

$$
A_{i}=A+k_{i} B C=\left(\begin{array}{cc}
a_{11} & a_{12} \\
a_{21}+k_{i} & a_{22}
\end{array}\right) .
$$

Since $a_{12} \neq 0$, if $a_{11}+a_{22}<0$, obviously there exists output feedback gain $k_{i}$ such that $A_{i}$ is Hurwitz stable, that is, system (13) could be static output feedback stabilizable. As for the case $a_{11}+a_{22} \geq 0$, let $\Delta_{i}$ is the discriminant of the characteristic equation for the coefficient matrices $A_{i}, i=1,2$, If we choose $k_{i}$ such that

$$
\Delta_{i}=4\left(\alpha^{2}-|A|+a_{12} k_{i}\right)<0
$$

then $A_{i}$ 's eigenvalues are express as $\lambda_{i \pm}=\alpha+j \beta_{i}$, where

$$
\begin{gathered}
\alpha=\frac{a_{11}+a_{22}}{2}, \\
\beta_{i}=\sqrt{|A|-\alpha^{2}-a_{12} k_{i}} .
\end{gathered}
$$

From Lemma 1, we know there exists a coordinate transformation $y=Q x$, $Q=\left(q_{i j}\right)_{2 \times 2}$, changes the closed-loop system into $\dot{y}=A_{i}^{\prime} y$, where $A_{i}^{\prime}=Q A_{i} Q^{-1}$, $i=1,2$, and

$$
A_{1}^{\prime}=\left(\begin{array}{cc}
\alpha & \beta_{1} / E \\
-E \beta_{1} & \alpha
\end{array}\right), \quad A_{2}^{\prime}=\left(\begin{array}{cc}
\alpha & \beta_{2} \\
-\beta_{2} & \alpha
\end{array}\right) \text {. }
$$


Solving the equation $Q A_{i}=A_{i}^{\prime} Q, i=1,2$, we obtain that $E=\frac{\beta_{1}}{\beta_{2}}, q_{12}=0$, and

$$
\begin{gathered}
\left(a_{11}-a_{22}\right) q_{22}=2 a_{12} q_{21}, \\
\beta_{2} q_{22}=a_{12} Q_{11} .
\end{gathered}
$$

If we further assume that

$$
\beta_{1}<\beta_{2},
$$

then according the discussion in Section 2, we have

$$
0<E<1, \quad D=\frac{1}{4}\left(E-\frac{1}{E}\right)^{2}>0
$$

and

$$
\rho=\prod_{i=1}^{2} \exp \rho_{i}\left(\arctan \rho_{i}-\frac{\pi}{2}\right) \times \sqrt{\frac{\alpha^{2}+\beta_{2}^{2}}{\alpha^{2}+\beta_{1}^{2}}} .
$$

Under the coordinate transformation $y=Q x$, we obtain that at the parallel lines

$$
\nu_{ \pm}^{\prime}=\frac{y_{2}}{y_{1}}=\frac{q_{21} x_{1}+q_{22} x_{2}}{q_{11} x_{1}+q_{12} x_{2}}
$$

that is,

$$
\nu_{ \pm}=\frac{q_{21}-\nu_{ \pm}^{\prime}}{q_{12} \nu_{ \pm}^{\prime}-q_{12}} .
$$

Therefore

$$
\rho_{1}-\frac{\rho_{2}}{E}=0, \quad \nu_{-}^{\prime}=\infty, \quad \nu_{+}^{\prime}=-\frac{\alpha}{\beta_{2}}
$$

In view of the discussion thus far, we should choose the constants $k_{i}$ satisfies (15), (18), and such that $\beta_{i}$ satisfies $\rho<1$. It is not difficult to show that such $k_{i}$ always exist. Therefore, we conclude that in this case system (13) always stabilizable via the 2-state hybrid output feedback control

$$
u=\left\{\begin{array}{cc}
k_{1} y \quad x_{2} \leq-\frac{a_{22}}{a_{12}} x_{1}, \text { and } x_{1} \geq 0, \text { or } \\
x_{2} \geq-\frac{a_{22}}{a_{12}} x_{1}, \text { and } x_{1} \leq 0 \\
\quad \text { otherwise }
\end{array}\right.
$$

The proof is complete.

Remark 2. For $c \neq 0$, and $a_{12} \neq 0$, we could also design the output feedback gains such that closed-loop switched system satisfies the condition of Theorem 1. Of course they might be nonexistence. When $a_{12}=0$, system (13) is uncontrollable, then unstabilizable. 


\section{A Numerical Example}

Example 2. Consider the linear system of type (13) with

$$
A=\left(\begin{array}{ll}
-1 & 2 \\
-2 & 3
\end{array}\right), \quad B=\left(\begin{array}{l}
0 \\
1
\end{array}\right), \quad C=\left(\begin{array}{ll}
1 & 0
\end{array}\right) .
$$

We can easily verify that the pair $\left(k_{1}, k_{2}\right)=(-1,-38)$ satisfies the required conditions (15), (18), and $\rho=0.8697<1$. Then the 2 -state hybrid output feedback stabilizable control is

$$
u=\left\{\begin{array}{cc}
-y & 0 \leq x_{1} \leq-\frac{2}{3} x_{2}, \text { or }-\frac{2}{3} x_{2} \leq x_{1} \leq 0 \\
-38 y & \text { otherwise }
\end{array} .\right.
$$

Fig. 2 shows the trajectory of the system with the initial condition $x(0)=(2,2)$.

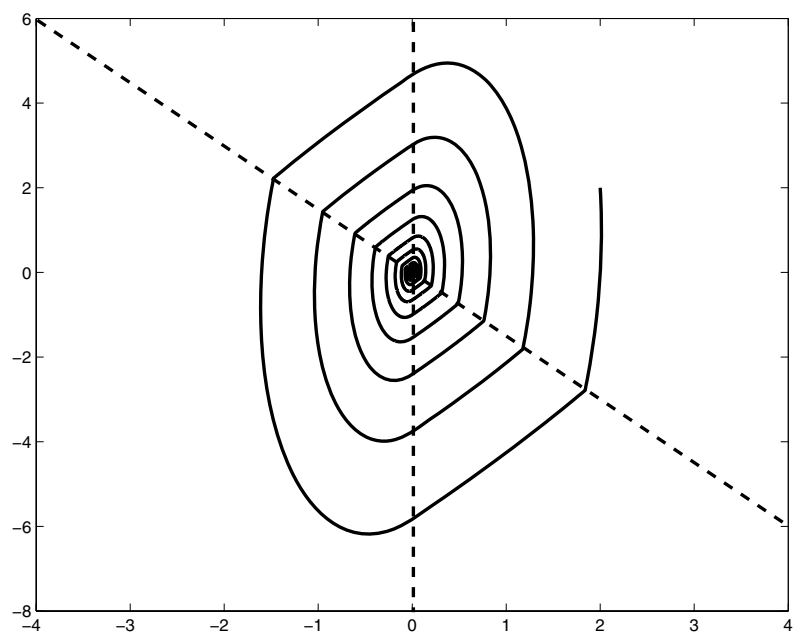

Fig. 2. The trajectory for Example 2

\section{Conclusion}

This paper studies the hybrid output feedback stabilization of second-order linear dynamic systems. For a class of such systems, we showed that the system is stabilizable via a 2 -state static output feedback incorporated with an appropriate switching law. Output feedback gains and switching law are constructed in terms of control system matrices. Extension of the proposed results to general highdimensional linear systems is considered to be an interesting future work.

\section{Acknowledgements}

This paper was supported in part by the National Natural Science Foundation of China (No.60374007), and in part by the Beijing Natural Science Foundation of China (No.4042006). 


\section{References}

1. Syrmos, V.L., Abdallah, C.T., Dorato, P., and Grigoriadis, K.: Static output feedback: a survey. Automatica. 33 (1997) 125-137

2. Artstein, Z.: Examples of stabilization with hybrid feedback, in: R. Alur et al. (Eds.), Hybrid Systems III: Verification and Control, (1996) 173-185

3. Liberzon, D.: Stabilizing a linear system with finite-state hybrid output feedback, in: Proceedings of the 7th IEEE Mediterranean Conference on Control and Automation, (1999) 176-183

4. Liberzon, D., Morse, A.S.: Basic problems in stability and design of switched systems, IEEE Control Systems Magazine 19 (1999) 59-70

5. Hu, B., Zhai, G., Michel, A.N.: Hybrid output feedback stabilization of twodimensional linear control systems, in: Proceedings of the 2000 American Control Conference, (2000) 2184-2188

6. Branicky, M.S.: Multiple Lyapunov functions and other analysis tools for switched and hybrid systems, IEEE Trans. Automat. Control 43 (1998) 475-482

7. DeCarlo, R., Branicky, M., Pettersson, S., Lennartson,B.: Perspectives and results on the stability and stabilizability of hybrid systems, Proc. IEEE 88 (2000) 10691082

8. Johansson, M., Rantzer, A.: Computation of piecewise quadratic Lyapunov functions for hybrid systems, IEEE Trans. Automat. Control 43 (1998) 555-559

9. Michel, A.N.: Recent trends in the stability analysis of hybrid dynamical systems, IEEE Trans. Circuit and Systems-I: Fundamental Theory and Applications 45 (1999) 120-134

10. Morse, A.S.: Control using logic-based switching, in: A. Isidori (Ed.), Trends in Control: a European Perspective, Springer, Berlin, (1995) 69-113

11. Peleties, P., DeCarlo, R.: Asymptotic stability of $\mathrm{m}$-switched systems using Lyapunov-like functions, in: Proceedings of the 1991 American Control Conference, (1991) 1679-1684

12. Litsyn, E., Nepomnyashchikh, Y.V., and Ponosov, A.: Stabilization of linear differential systems via hybrid feedback controls, SIAM J. Control Optim. 38 (2000) $1468-1480$

13. Xu, X., Antsaklis, P.J.: Design of stabilizing control laws for second-order switched systems, in: Proceedings of the 14th IFAC World Congress, vol. C, (1999) 181-186

14. Xu, X., Antsaklis, P.J.: Stabilization of second-order LTI switched systems, Int. J. Control 73 (2000) 1261-1279

15. Sastry, S.: Nonlinear Systems, Springer, New York, (1999)

16. Zhang, L., Chen, Y., and Cui, P.: Stabilization of a Class of Switched Linear Systems, Nonlinear Analysis: Theory, Methods and Applications, Special Issue on Hybrid Systems and Applications, 62 (2005) 1527-1535 